\title{
Heterozygosity at the malate dehydrogenase locus and developmental homeostasis in Apis mellifera
}

\author{
SAMANTHA MESSIER \& JEFFRY B. MITTON* \\ Department of Environmental, Population, and Organismic Biology, Box 334, University of Colorado, Boulder, CO \\ 80309-0334, U.S.A.
}

\begin{abstract}
We compared fluctuating asymmetry (FA) of wing vein lengths in honeybees (Apis mellifera) between heterozygotes and homozygotes at the malate dehydrogenase $(M D H)$ locus. FA is an indirect measure of developmental homeostasis - the ability of individuals to develop normal phenotypes despite genetic and environmental stresses. Heterozygosity is thought to have a positive influence on developmental homeostasis; hence we hypothesized that honeybees heterozygous for the $M D H$ locus would have greater developmental homeostasis and less FA than homozygotes. Our data support this hypothesis. For one of the five characters measured, $M D H$ heterozygotes displayed less FA than homozygotes in both colonies we examined. We also detected differences in character size and character correlation between $M D H$ heterozygotes and homozygotes, although these patterns were not consistent across colonies. A discriminant function analysis of wing vein characters revealed significant differences between homozygotes and heterozygotes.
\end{abstract}

Keywords: Apis mellifera, developmental homeostasis, fluctuating asymmetry, heterozygosity.

\section{Introduction}

Developmental homeostasis is the ability of individuals to develop phenotypes within the normal range of expression despite environmental and genetic perturbations (Lerner, 1954). Some individuals may have greater homeostatic capacity than others and therefore should exhibit morphological phenotypes closer to the population mean. One form of morphological variation particularly useful to students of developmental homeostasis is fluctuating asymmetry (FA) - uncorrelated differences between paired characters on either side of a bilaterally symmetrical organism (Van Valen, 1962). Paired characters on either side of an organism should be under the control of the same developmental mechanism and should reach identical proportions unless perturbations disrupt canalization. FA is assumed to be negatively correlated with the capacity for developmental homeostasis.

Two nonmutually exclusive hypotheses for genetic influence on developmental homeostasis are genetic

${ }^{*}$ Correspondence. coadaptation and heterozygosity. Genetic coadaptation results from the balance of alleles both within and among chromosomes and is thought to be maintained by stabilizing selection (Mather, 1973; Clarke et al., 1992; Clarke, 1993). Several recent studies offer evidence for the influence of genetic coadaptation on developmental homeostasis (reviewed by Clarke, 1993; McKenzie \& O'Farrell, 1993). Moreover, there is a great deal of evidence demonstrating a positive relationship between heterozygosity and various measures of developmental homeostasis (Mitton \& Koehn, 1975; Brückner, 1976; Eanes, 1978; Mitton, 1978; Soulé, 1979; Leary et al., 1983, 1985; King, 1985). In many cases, especially those involving inbred populations, it has been difficult to sort out the influences of heterozygosity and genetic coadaptation. In the case of Soulés $(1967,1979)$ work on lizard populations, his original conclusion that developmental homeostasis resulted from genic balance was amended to express the conclusion that heterozygosity alone could have accounted for much of the variation in FA. We stress the fact that there is room for both of these mechanisms - heterozygosity and genetic coadaptation - to operate within individuals. 
Haplodiploid organisms provide a particularly interesting system in which to examine the effect of heterozygosity on developmental stability. Because reproductive drones are haploid, heterozygosity cannot influence developmental stability in males. Nevertheless, increased heterozygosity in females may give them added buffering capacity as well as influence differences in developmental homeostasis between females. Previous studies of developmental homeostasis in honeybees have produced conflicting results. Comparing inbred and outbred stocks, Brückner (1976) found that highly heterozygous workers were less morphologically variable than highly homozygous workers and that workers in general were less variable than hemizygous drones. In contrast, Clarke et al. (1992) found no significant relationship between degree of inbreeding and FA. Honeybees are already highly homozygous because of haplodiploidy and other factors (Lester \& Selander, 1979; Avery, 1984). Therefore it may be difficult to detect differences between inbred and outbred colonies. We chose to avoid this problem by choosing one enzyme locus and assessing the relationship between heterozygosity at this locus and developmental homeostatis as revealed by the level of FA.

Honeybees show very little genetic variation within populations and are monomorphic at most enzyme loci (Pamilo et al., 1978). Malate dehydrogenase $(M D H)$ is an unusual exception to this pattern in that it is polymorphic in most honeybee populations (Badino et al., 1983). Furthermore, Coelho \& Mitton (1988) demonstrated a relationship between $M D H$ genotype and maximal metabolic rate in honeybees. Because of its consistent polymorphism and relationship to physiological efficiency, we chose the $M D H$ locus for our comparison of FA among genotypes. We hypothesized that individuals heterozygous for the $M D H$ locus would display less FA than individuals homozygous at this locus.

\section{Methods}

\section{Collection}

We collected $\approx 100$ mature foragers of Apis mellifera ligustica from each of two separate colonies maintained in Boulder County, Colorado. Workers from colony one were collected in September 1991. Workers from colony two were collected in September 1993. Bees were collected by removing them one at a time from the hive entrance as they returned from or were departing on foraging trips. The bees were frozen within $1 \mathrm{~h}$ of collection and stored at $-20^{\circ} \mathrm{C}$.

\section{Electrophoresis}

We removed the head of each bee and pulverized it in $0.2 \mathrm{~mL}$ dI $\mathrm{H}_{2} \mathrm{O}$. We centrifuged the resulting homogenate in a clinical centrifuge for one minute and extracted the supernatant for use in horizontal starch gel electrophoresis. The samples were subjected to $50 \mathrm{~mA}$ and $150 \mathrm{mV}$ for $4 \mathrm{~h}$ at $4^{\circ} \mathrm{C}$ using the buffer system described by Tanaka et al. (1980). The gels were then stained for MDH activity (Shaw \& Prasad, 1969).

\section{Morphometric measurements}

For each bee we measured wet weight, without the head. We then removed the forewings from the thorax and mounted them on glass slides. We designated five wing veins to be examined for fluctuating asymmetry. We mounted the glass slides on a photographic enlarger and measured the length of each vein in the enhanced image with callipers (Fig. 1). The length of the enlarged image of the vein was measured in millimetres. The length of the glass cover slip on each slide was also measured to check that the amount of enlargement did not vary. As a blind, measurements were taken without previous knowledge of each individual's $M D H$ genotype. Some of the wings were damaged during mounting; hence we were unable to measure every character for each bee.

\section{Statistical analysis}

As indices of FA, we used both $\Sigma\left|R_{i}-L_{i}\right| / N$ and $\Sigma\left(R_{i}-L_{i}\right)^{2} / N$ (Palmer \& Strobeck, 1986). For each

4

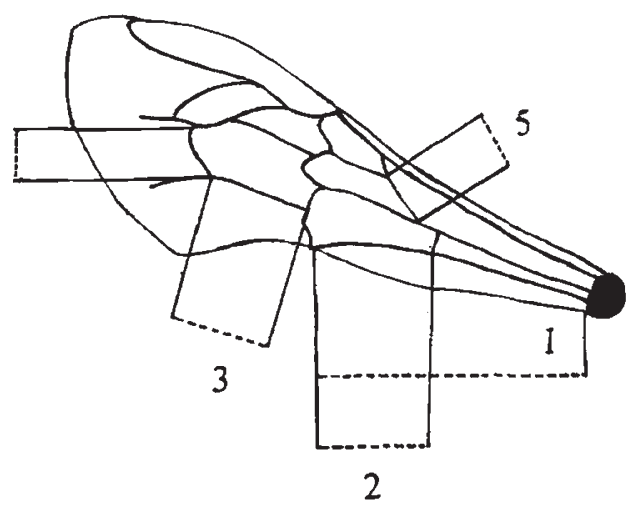

Fig. 1 Characters measured on forewings of Apis mellifera. 
index, we compared fluctuating asymmetry between heterozygotes and homozygotes by ANOVA. The test was one-tailed, with the expectation that homozygotes should display greater FA for the characters measured. We also examined the possibility that wings of different genotypes at this locus might differ in shape. We compared mean vein lengths for each wing vein among $M D H$ genotypes by ANOVA. We also tested whether correlation coefficients between wing vein lengths varied among genotypes using a test of homogeneity among correlation coefficients (Sokal \& Rohlf, 1969). We also performed a direct discriminant function analysis using the five wing vein length characters, separating bees into heterozygotes and homozygotes at the $\mathrm{MDH}$ locus. Missing values for individual characters were excluded.

\section{Results}

\section{Genotypic frequencies}

Electrophoresis revealed two $\mathrm{MDH}$ allozymes in the colonies, fast $(F)$ and slow $(S)$. The $M$ allozyme was not found in either of the two colonies. Of the 96 bees from colony one, 61 were $F S$ heterozygotes and 35 were $S S$ homozygotes. There were no $F F$ homozygotes. Of the 106 bees from colony two, 45 were $F S$ heterozygotes, 34 were $F F$ homozygotes, and 27 were $S S$ homozygotes.

\section{Character size}

In colony one, four of the wing veins (L1, L4, R1 and R4) showed differences in length between heterozygotes and homozygotes (Table 1a). No such differences were found in colony two (Table 1b). In each of the cases where differences were found, measurements for heterozygotes were larger than measurements for homozygotes. Heterozygotes had a mean weight of $82.8 \mathrm{mg}$. The mean weight for homozygotes was $84.0 \mathrm{mg}$. These means were not different by a $t$-test $\left(t_{90}=-0.897, P>0.3\right)$. This would imply that the differences in wing vein length between heterozygotes and homozygotes are not necessarily simply a matter of heterozygotes being larger. The comparison of correlation coefficients and the discriminant function analysis support this interpretation.

Table 1 Mean lengths in $\mathrm{mm} \pm \mathrm{SE}$, of wing veins of Apis mellifera measured from an enlarged image (with sample sizes in parentheses)

\begin{tabular}{lrrrr}
\hline Wing vien & \multicolumn{1}{c}{$F S$} & \multicolumn{1}{c}{$S S$} & $F F$ & $P$ \\
\hline (a) Colony 1 & & & & \\
L1 & $27.965 \pm 0.070(53)$ & $27.624 \pm 0.090(29)$ & - & 0.0001 \\
L2 & $11.608 \pm 0.038(59)$ & $11.580 \pm 0.050(33)$ & - & 0.6891 \\
L3 & $10.212 \pm 0.036(60)$ & $10.233 \pm 0.045(32)$ & - & 0.7656 \\
L4 & $5.083 \pm 0.031(59)$ & $4.936 \pm 0.035(33)$ & - & 0.0375 \\
L5 & $5.466 \pm 0.037(55)$ & $5.377 \pm 0.061(33)$ & - & 0.0058 \\
R1 & $27.948 \pm 0.059(53)$ & $27.741 \pm 0.096(29)$ & - & 0.9358 \\
R2 & $11.618 \pm 0.040(59)$ & $11.612 \pm 0.068(33)$ & - & 0.3870 \\
R3 & $10.206 \pm 0.036(60)$ & $10.267 \pm 0.048(32)$ & - & 0.0063 \\
R4 & $5.117 \pm 0.033(59)$ & $4.924 \pm 0.035(33)$ & & 0.5578 \\
R5 & $5.437 \pm 0.039(55)$ & $5.395 \pm 0.039(33)$ & & 0.2941 \\
(b) Colony 2 & & & & 0.5570 \\
L1 & $18.328 \pm 0.046(41)$ & $18.211 \pm 0.062(24)$ & $7.818 \pm 0.030(33)$ & 0.0639 \\
L2 & $7.781 \pm 0.026(45)$ & $7.767 \pm 0.041(26)$ & $6.963 \pm 0.031(33)$ & 0.4205 \\
L3 & $6.999 \pm 0.039(45)$ & $6.87 \pm 0.037(26)$ & $3.331 \pm 0.030(33)$ & 0.1327 \\
L4 & $3.299 \pm 0.026(44)$ & $3.352 \pm 0.030(26)$ & $3.884 \pm 0.038(34)$ & 0.2114 \\
L5 & $3.864 \pm 0.036(45)$ & $3.768 \pm 0.043(25)$ & $18.311 \pm 0.053(29)$ & 0.4228 \\
R1 & $18.350 \pm 0.045(41)$ & $18.213 \pm 0.060(24)$ & $7.777 \pm 0.031(33)$ & 0.0963 \\
R2 & $7.746 \pm 0.022(45)$ & $7.726 \pm 0.029(26)$ & $7.020 \pm 0.039(33)$ & 0.4841 \\
R3 & $6.969 \pm 0.033(45)$ & $6.892 \pm 0.043(26)$ & $3.340 \pm 0.038(33)$ & 0.4758 \\
R4 & $3.305 \pm 0.032(44)$ & $3.363 \pm 0.030(26)$ & $3.844 \pm 0.038(33)$ & -050 \\
R5 & $3.873 \pm 0.033(45)$ & $3.809 \pm 0.048(25)$ & & - \\
\hline
\end{tabular}

$\mathrm{L}$ indicates left wing, $\mathrm{R}$ indicates right wing. Larger measurements for colony 1 are the result of greater enlargement. Means are compared by ANOVA and Fisher's PLSD. 


\section{Fluctuating asymmetry}

Homozygotes at the $M D H$ locus had higher levels of FA for wing vein 2 in both colonies. In colony one, $\Sigma\left|R_{i}-L_{i}\right| / N$ was significantly less for heterozygotes than for homozygotes (Table 2a). In colony two, $\Sigma\left(R_{i}-L_{i}\right)^{2} / N$ was significantly less for heterozygotes than for pooled homozygotes (Table $2 b$ ).

\section{Correlation analysis}

In colony one, the correlations between R3 and R5 were significantly different between heterozygotes and homozygotes $\left(\chi_{1}^{2}=8.59, P<0.01\right)$ (Table 3 ). This difference suggests that the wings of hetero- zygotes and homozygotes in colony one had different shapes. In colony two, the pattern of correlations is more complex because of the presence of three genotypes. The correlation between R2 and R3 is different between heterozygotes and pooled homozygotes and between heterozygotes and $F F$ homozygotes; however, it is not different between heterozygotes and $S S$ homozygotes or between $F F$ homozygotes and SS homozygotes (Table 4). The correlation between R2 and R4 follows the same pattern. The correlation between R3 and R4 differs between the two homozygotes, but it does not differ between either homozygote and heterozygotes (Table 4). Again, these relationships suggest a

Table 2 Mean fluctuating asymmetry (FA) $\pm \mathrm{SE}$ for wing veins of Apis mellifera (wing sample sizes in parentheses)

\begin{tabular}{|c|c|c|c|c|c|c|}
\hline \multirow{2}{*}{$\begin{array}{l}\text { Wing } \\
\text { vein }\end{array}$} & \multicolumn{3}{|c|}{ Colony 1} & \multicolumn{3}{|c|}{ Colony 2} \\
\hline & Heterozygotes & Homozygotes & $F$ & Heterozygotes & Homozygotes & $F$ \\
\hline \multicolumn{7}{|c|}{ (a) $\mathrm{FA}=\left|L_{i}-R_{i}\right|$} \\
\hline 1 & $0.309 \pm 0.037(53)$ & $0.314 \pm 0.052(29)$ & 0.005 & $0.198 \pm 0.027(45)$ & $0.157 \pm 0.023(60)$ & 1.138 \\
\hline 2 & $0.150 \pm 0.030(53)$ & $0.205 \pm 0.017$ & $2.859^{*}$ & $0.135 \pm 0.014(45)$ & $0.167 \pm 0.016(60)$ & 2.055 \\
\hline 3 & $0.179 \pm 0.017(60)$ & $0.163 \pm 0.022(32)$ & 0.344 & $0.161 \pm 0.018$ & $0.165 \pm 0.018(59)$ & 0.027 \\
\hline 4 & $0.131 \pm 0.013(59)$ & $0.152 \pm 0.021(33)$ & 0.795 & $0.156 \pm 0.017(45)$ & $0.149 \pm 0.016(61)$ & 0.100 \\
\hline 5 & $0.207 \pm 0.022(55)$ & $0.200 \pm 0.039$ & 0.030 & $0.178 \pm 0.021(45)$ & $0.151 \pm 0.015(61)$ & 1.160 \\
\hline \multicolumn{7}{|c|}{ (b) $\mathrm{FA}=\left(L_{i}-R_{i}\right)^{2}$} \\
\hline 1 & $0.166 \pm 0.040$ & $0.175 \pm 0.065(29)$ & 0.015 & $0.072 \pm 0.018$ & $0.055 \pm 0.018(61)$ & 0.447 \\
\hline 2 & $0.040 \pm 0.009(59)$ & $0.070 \pm 0.021$ & 2.359 & $0.027 \pm 0.005$ & $0.043 \pm 0.007(60)$ & $2.844^{*}$ \\
\hline 3 & $0.050 \pm 0.009(60)$ & $0.041 \pm 0.009$ & 0.348 & $0.040 \pm 0.008$ & $0.047 \pm 0.010(59)$ & 0.307 \\
\hline 4 & $0.027 \pm 0.005$ & $0.037 \pm 0.008$ & 1.116 & $0.037 \pm 0.007$ & $0.037 \pm 0.007(61)$ & 0.010 \\
\hline 5 & $0.070 \pm 0.013(55)$ & $0.088 \pm 0.047$ & 0.208 & $0.052 \pm 0.013$ & $0.036 \pm 0.006(62)$ & 1.438 \\
\hline
\end{tabular}

Means for homozygotes and heterozygotes compared by ANOVA (one-tailed, expecting homozygotes to be larger).

${ }^{*} P<0.05$.

Table 3 Matrices of Pearson correlation coefficients for wing vein lengths for colony 1 of Apis mellifera

\begin{tabular}{|c|c|c|c|c|c|}
\hline & $\mathrm{R} 1$ & $\mathrm{R} 2$ & R3 & $\mathrm{R} 4$ & R5 \\
\hline \multicolumn{6}{|c|}{ Heterozygotes } \\
\hline $\mathrm{R} 1$ & 1.000 & $0.559^{*}$ & 0.110 & $0.367^{*}$ & $0.377^{*}$ \\
\hline $\mathrm{R} 2$ & & 1.000 & 0.248 & $0.405^{*}$ & $0.321^{*}$ \\
\hline R3 & & & 1.000 & 0.056 & $0.471^{*} \dagger$ \\
\hline $\mathrm{R} 4$ & & & & 1.000 & $0.281^{*}$ \\
\hline \multicolumn{6}{|c|}{ Homozygotes } \\
\hline R1 & 1.000 & $0.702^{*}$ & 0.013 & 0.289 & 0.235 \\
\hline $\mathrm{R} 2$ & & 1.000 & -0.053 & $0.375^{*}$ & $0.469^{*}$ \\
\hline R3 & & & 1.000 & -0.324 & $-0.205 \dagger$ \\
\hline $\mathrm{R} 4$ & & & & 1.000 & 0.260 \\
\hline
\end{tabular}

*Different from zero, $P<0.05$; †different between heterozygotes and homozygotes. 
Table 4 Matrices of Pearson correlation coefficients for wing vein lengths for colony 2 of Apis mellifera

\begin{tabular}{|c|c|c|c|c|c|}
\hline & $\mathrm{R} 1$ & $\mathrm{R} 2$ & R3 & $\mathrm{R} 4$ & R5 \\
\hline \multicolumn{6}{|c|}{ Heterozygotes } \\
\hline R1 & 1.000 & 0.120 & 0.115 & -0.134 & -0.211 \\
\hline R2 & & 1.000 & $0.358^{*} \dagger \neq$ & $-0.119 \dagger$ & 0.061 \\
\hline R3 & & & 1.000 & -0.134 & 0.003 \\
\hline R4 & & & & 1.000 & 0.226 \\
\hline \multicolumn{6}{|c|}{$F F$ and $S S$ homozygotes pooled } \\
\hline $\mathrm{R} 1$ & 1.000 & 0.137 & 0.133 & 0.076 & 0.186 \\
\hline R2 & & 1.000 & $0.091 \S$ & $0.349 * \S$ & 0.229 \\
\hline R3 & & & 1.000 & -0.050 & -0.109 \\
\hline R4 & & & & 1.000 & $0.302^{*}$ \\
\hline \multicolumn{6}{|c|}{$F F$ homozygotes } \\
\hline $\mathrm{R} 1$ & 1.000 & 0.139 & -0.005 & -0.039 & 0.020 \\
\hline $\mathrm{R} 2$ & & 1.000 & $-0.047 \S$ & $0.393 * \S$ & 0.181 \\
\hline R3 & & & 1.000 & $-0.238 \pi$ & -0.232 \\
\hline R4 & & & & 1.000 & 0.204 \\
\hline \multicolumn{6}{|c|}{$S S$ homozygotes } \\
\hline $\mathrm{R} 1$ & 1.000 & 0.039 & 0.169 & 0.318 & 0.283 \\
\hline $\mathrm{R} 2$ & & 1.000 & 0.174 & 0.301 & 0.269 \\
\hline R3 & & & 1.000 & $0.377 \ddagger$ & -0.110 \\
\hline $\mathrm{R} 4$ & & & & 1.000 & $0.488^{*}$ \\
\hline
\end{tabular}

${ }^{*}$ Different from zero, $P<0.05$; $\dagger$ different from $F F$ and $S S$ homozygotes pooled; łdifferent from $F F$ homozygotes; §different from heterozygotes; Idifferent from SS homozygotes.

difference in shape among genotypes, although the pattern differs from that in colony one.

\section{Discriminant function analysis}

In colony one, a direct discriminant function analysis correctly classified 73 per cent of the individuals as either homozygotes or heterozygotes based on the five wing vein characters used in this study $(P \leq 0.02)$. Wing vein characters $\mathrm{R} 1$ and $\mathrm{R} 4$, which showed significant differences in size between heterozygotes and homozygotes, were also the most useful characters for the discriminant function analysis (Table 5). In colony two, the analysis correctly classified 61 per cent of individuals as either homozygotes or heterozygotes (pooled $F F$ and $S S$ ) $(P \leq 0.39)$. The most useful characters for discrimiination in this colony were R4 and R5 (Table 5). When the three genotypes in colony two were grouped separately, the analysis correctly identified 45 per cent of individuals by $M D H$ genotype $(P \leq 0.39)$.
Table 5 Standardized canonical discriminant function coefficients distinguishing wings of $M D H$ heterozygotes and homozygotes of Apis mellifera

\begin{tabular}{lrr}
\hline \multirow{2}{*}{$\begin{array}{l}\text { Wing } \\
\text { vein }\end{array}$} & \multicolumn{2}{c}{ Discriminant function coefficient } \\
\cline { 2 - 3 } & Colony 1 & Colony 2 \\
\hline R1 & 0.415 & 0.250 \\
R2 & -0.392 & -0.144 \\
R3 & -0.321 & 0.273 \\
R4 & 0.768 & -0.892 \\
R5 & 0.239 & 0.791 \\
\hline
\end{tabular}

\section{Discussion}

Fluctuating asymmetry is greater in homozygotes than in heterozygotes at the $M D H$ locus for at least one wing vein character in honeybees. This finding supports the hypothesis that heterozygosity contributes to developmental homeostasis in this haplodiploid species. We acknowledge that other factors, including genetic coadaptation, probably also contri- 
bute to developmental homeostasis in honeybees, but these data clearly demonstrate a role for heterozygosity. The argument for the importance of heterozygosity in this case is especially convincing given that a within-population difference in FA could be detected between heterozygotes and homozygotes at a single polymorphic enzyme locus. It is not surprising that only one character out of five showed differences in FA. Symmetry of wing veins in honeybees may be under stabilizing selection, which could obscure differences in FA.

It is unclear as to whether it is heterozygosity at the $M D H$ locus or at a linked locus which appears to enhance developmental homeostasis. The possibility also exists that $M D H$ might serve as an index of total heterozygosity, although we believe this to be unlikely. The bees used in this study were from colonies with naturally mated queens. Honeybee queens typically mate with 7-17 males (Winston, 1987). Therefore, $M D H$ genotype for any daughter bee results from the inheritance of one allele from the queen and one allele from any of these many possible haploid males. This significantly reduces the possibility that $M D H$ genotype in every worker is linked to the same entire set of chromosomes inherited from the male.

The results of this study also suggest that not only do heterozygotes at the $M D H$ locus exhibit greater developmental homeostasis, but genotype at this locus or a locus in linkage disequilibrium with it may directly influence development. This finding is consistent with other research demonstrating an effect of allozymes on development. DiMichele et al. (1991) and Paynter et al. (1991) demonstrated that LDH (lactate dehydrogenase) allozymes have an effect on the development of killifish. Leary et al. (1993) examined the influence of a null allele at the LDH locus and revealed that heterozygotes for a null allele had greater fluctuating asymmetry than homozygotes for an active allele. They concluded that they were measuring the developmental consequences of genetic variation at a single locus.

Based on our comparison of wing vein lengths between heterozygotes and homozygotes, it would appear that heterozygotes possess a different wing shape from homozygotes. For colony one, the data on wing vein size alone may suggest that heterozygotes are merely larger overall. However, the weights of heterozygotes and homozygotes did not differ, suggesting no difference in overall size between the two. Also, colony two did not exhibit these size differences. Several characters were correlated differently in homozygotes than in heterozygotes - implying that heterozygotes and (c) The Genetical Society of Great Britain, Heredity, 76, 616-622. homozygotes do indeed differ in shape. Again, the pattern was different for the two colonies. Perhaps the most compelling evidence for a difference in developmental outcome is the result of the discriminant function analysis. This analysis was able to classify correctly individuals as heterozygotes or homozygotes in 73 per cent $(P<0.02)$ of cases for colony. one and 61 per cent $(P<0.39)$ of cases for colony two. We believe these results suggest a discernible, although inconsistent, effect of $\mathrm{MDH}$ allozymes on development in honeybees.

\section{Acknowledgements}

We thank T. Arnett, A. Maruyama and K. Scott for their assistance in measuring wing veins, S. Wooding for assistance collecting worker bees, and M. D. Breed for the use of his honeybee hives.

\section{References}

AVERY, P. J. 1984. The population genetics of haplodiploids and X-linked genes. Genet. Res., 44, 321-341.

Badino, F., Celebrano, F. AND manino, A. 1983. Population structure and MDH-1 locus variation in Apis mellifera ligustica. J.Hered., 74, 443-446.

BRÜCKNER, D. 1976. The influence of genetic variability on wing symmetry in honeybees. (Apis mellifera). Evolution, 30, 100-108.

CLARKE, G. M. 1993. The genetic basis of developmental stability. I. Relationships between stability, heterozygosity, and genomic coadaptation. Genetica, 89, 15-23.

ClARKE, G. M., OLDROYD, B. P. AND hUNT, P. L. 1992. The genetic basis of developmental stability in Apis mellifera: heterozygosity versus genic balance. Evolution, 46, $753-762$.

COELHO, J. R. AND MITTON, J. B. 1988. Oxygen consumption during hovering is associated with genetic variation of enzymes in honey-bees. Funct. Ecol., 2, 141-146.

DIMICHELE, L., PAYNTER, K. T. AND POWERS, D. A. 1991. Evidence of lactate dehydrogenase-B allozyme effects in the teleost, Fundulus heteroclitus. Science, 253, 898-900.

EANES, W. F. 1978. Morphological variance and enzyme heterozygosity in the monarch butterfly. Nature, 276, 263-264.

KING, D. P. F. 1985. Enzyme heterozygosity associated with anatomical character variance and growth in the herring (Clupea harengus L.). Heredity, 54, 289-296.

LEARY, R. F., ALLENDORF, F. W. AND KNUDSEN, K. L. 1983. Developmental stability and enzyme heterozygosity in rainbow trout. Nature, 301, 71-72.

LEARY, R. F., ALLENDORF, F. W. AND KNUDSEN, K. L. 1985. Inheritance of meristic variation and the evolution of developmental stability in rainbow trout. Evolution, 39, 308-314. 
LEARY, R. F., ALLENDORF, F. W. AND KNUDSEN, K. L. 1993. Null alleles at two lactate dehydrogenase loci in rainbow trout are associated with decreased developmental stability. Genetica, 89, 3-13.

LERNer, 1. M. 1954. Genetic Homeostasis. Oliver and Boyd, London.

LESTER, L. J. AND SElANDER, R. K. 1979. Population genetics of haplo-diploid insects. Genetics, 92, 1329-1345.

McKenziE, J. A. AND o'farrell, K. 1993. Modification of developmental instability and fitness: malathion-resistance in the Australian sheep blowfly, Lucilia cuprina. Genetica, 89, 67-76.

MAther, K. 1973. Genetical Structure of Populations. Chapman and Hall, London.

MitTon, J. B. 1978. Relationship between heterozygosity for enzyme loci and variation of morphological characters in natural populations. Nature, 273, 661-662.

MITTON, J. B. AND KOEHN, R. K. 1975. Genetic organization and adaptive response of allozymes to ecological variables in Fundulus heteroclitus. Genetics, 79, 97-111.

PALMER, A. R. AND STROBECK, C. 1986. Fluctuating asymmetry: measurement, analysis, patterns. Ann. Rev. Ecol. Syst., 17, 392-421.

PAMILO, P., VARVIO-AHO, S. AND PEKKARINEN, A. 1978. Low enzyme variability in Hymenoptera as a consequence of haplo-diploidy. Hereditas, 88, 93-99.

PAYNTER, K. T., DIMICHELE, L., HAND, S. C. AND POWERS, D. 1992. Metabolic implications of $L d h-B$ genotype during early development in Fundulus heteroclitus. J. Exp. Zool., 257, 24-33.

SHAW, C. R. AND PRASAD., R. 1969. Starch gel electrophoresis of enzymes - a compilation of recipes. Biochem. Genet., 4, 45-48.

SOKAL, R. R. AND ROHLF, F. J. 1969. Biometry. W. H. Freeman and Co., San Francisco, CA.

SOULÉ, M. E. 1967. Phenetics of natural populations. II. Asymmetry and evolution in a lizard. Am. Nat., 101, $141-160$.

SOULÉ, M. E. 1979. Heterozygosity and developmental stability: another look. Evolution, 33, 396-401.

TANAKA, K., KUROSAWA, Y., KUROKAWA, K. AND OISHI, T. 1980. Genetic polymorphism of erythrocyte esterase-D in pigs. Animal Blood Groups and Biochem. Genet., 11, 193-197.

winston, M. L. 1987. The Biology of the Honey Bee. Harvard University Press, Cambridge, MA.

VAN VALEN, L. 1962. A study of fluctuating asymmetry. Evolution, 16, 125-142. 